Alicia Canetti, * M. Alejandra Girona Gamarra, ** Ana Cerutti» y lanina Tuñón*»

\title{
Construcción de una herramienta de medición del crecimiento, desarrollo y bienestar infantil: la experiencia del Centro Interdisciplinario de Infancia y Pobreza (CIIP)
}

\section{Construction of a tool for measuring the growth, development and child welfare: The experience of the Interdisciplinary Center for Children and Poverty (CIIP)}

Resumen | En las últimas décadas, en la mayoría de los países de la región, se ha avanzado en el reconocimiento del niño/a como sujeto de derechos y en la construcción de sistemas integrales de protección de la infancia, desplegándose políticas y programas destinados a la mejora de su bienestar y de la calidad del cuidado infantil. Estos esfuerzos requieren de medidas para evaluar el impacto real que producen y para analizar cómo se mueven las brechas de desigualdad en el tiempo. Este trabajo intenta resumir el proceso de construcción interdisciplinario de un sistema de indicadores multidimensional de medición del crecimiento, desarrollo integral y bienestar infantil, SINADIBI, elaborado por el Centro Interdisciplinario Infancia y Pobreza-EI-UDELAR. Asimismo, se presentan algunos resultados preliminares de su aplicación, ilustrando cómo la investigación académica puede contribuir en la construcción de estadísticas públicas confiables, que sirvan para la rendición de cuentas a la ciudadanía, orientar hacia un uso efectivo de la inversión pública y propiciar

\footnotetext{
Recibido: 1 de junio de 2017. Aceptado: 31 de julio de 2017.

* Responsable del Centro Interdisciplinario de Infancia y Pobreza (CIIP)-EI-UDELAR. Docente de la Facultad de Medicina. Médico psiquiatra con subespecialidad en psiquiatría de enlace. $*$ Centro Interdiciplinario Infancia y Pobreza. Escuela de Nutrición. Licenciada en nutrición. Magister en psicología social.

- Integrante del Centro Interdisciplinario de Infancia y Pobreza. Ex profesora adjunta de la licenciatura de psicomotricida, EUTM-Facultad de Medicina-UDELAR Maestra. Licenciada en psicomotricidad. Maestranda en salud comunitaria, Facultad de Enfermería-UDELAR. Ex directora del Plan CAIF-INAU en el periodo 2006-2010.

- Observatorio de la Deuda Social Argentina (UCA). Doctora en ciencias sociales. Magister en investigación en ciencias sociales. Licenciada en sociología (UBA). Asesora del CIIP.

Correos electrónicos: lancan@adinet.com.uy | alegirona2@gmail.com |

anacerutti35@gmail.com | ianina_tunon@uca.edu.ar
}

Canetti, Alicia et al. «Construcción de una herramienta de medición del crecimiento, desarrollo y bienestar infantil: la experiencia del Centro Interdisciplinario de Infancia y Pobreza (CIIP).» Interdisciplina 5, $\mathrm{n}^{\circ} 13$ (septiembre-diciembre 2017): 19-43. 
debates sobre integración social y efectivo ejercicio de derechos. El desafío es integrar la herramienta a un sistema único nacional de evaluación en Uruguay y emplearla en estudios colaborativos regionales, ofreciendo una mirada más amplia y comparada de la situación de la infancia en América Latina.

Abstract | In recent decades, in most countries of the region, progress has been made in the recognition of the child as a subject of rights and in the construction of comprehensive systems for the protection of children, with policies and programs aimed at improving their well-being and the quality of child care. These efforts require measures to assess the real impact they produce and to analyze how inequality gaps move over time. This paper attempts to summarize the process of interdisciplinary construction of a multidimensional tool for measuring growth, integral development and child wellbeing, SINADIBI, elaborated by the Interdisciplinary Center for Childhood and Poverty-EI-Udelar. Some preliminary results of its application are also presented, illustrating how academic research can contribute to the construction of reliable public statistics, which serve as accountability to the public, guide towards an effective use of public investment and promote debates on social integration and effective exercise of rights. The challenge is to integrate the tool into a single national evaluation system in Uruguay and to use it in regional collaborative studies, offering a broader and comparative view of the situation of children in Latin America.

Palabras clave | bienestar infantil, medición, pobreza, fragmentación social

Key Words | children well-being, measure, poverty, social fragmentation

\section{Introducción}

LA COMUNIDAD INTERNACIONAL reconoce el imperativo del desarrollo humano y social en numerosos instrumentos normativos. En la mayoría de los países de la región se ha progresado en el marco legal y en la adopción de sistemas integrales de protección de la infancia.

En las últimas décadas, los países de la región implementaron programas de transferencia de ingresos con una particular orientación a las familias y las infancias.

Igualmente se avanzó en la construcción de subsistemas de protección integrales focalizados en la primera infancia. A través de estos sistemas, los Estados asumen responsabilidades en la provisión de cuidado infantil y reconocen al niño ${ }^{1}$ como sujeto de derechos.

1 Con el propósito de evitar reiteradas aclaraciones en términos de género, se enfatiza que la referencia a "niños" incluye tanto a varones como a mujeres. 
Una pieza clave de los procesos de construcción de las políticas, programas y sistemas de protección es el acceso a información, evaluación y monitoreo. Sobre este aspecto también las situaciones de los países son diferentes. Justamente, en los últimos años se han desarrollado propuestas específicas de medición de la pobreza infantil desde un enfoque de derechos como la de CEPAL/ UNICEF (2012) que representa una importante contribución en términos de interpelar sobre la necesidad de construir datos que permitan una aproximación al estado de situación de poblaciones especialmente vulnerables como la infancia.

En este contexto se inscribe la propuesta de desarrollo de un Sistema Nacional Informatizado de Desarrollo Integral y Bienestar de la Infancia (SINADIBI) en el marco del Centro Interdisciplinario de Infancia y pobreza (CIIP) del Espacio Interdisciplinario-UDELAR, de Uruguay. La misma pretende ser un aporte para la evaluación y seguimiento del desarrollo de la infancia entre 0 y 7 años, ${ }^{2}$ residente en el país.

Medir el bienestar de los niños es una tarea compleja. Existe mucha discusión acerca de qué se incluye en esta noción, cuáles son sus dimensiones y cómo operacionalizarlas, por lo que se requiere contar con definiciones y modelos integrales consensuados a partir de la discusión interdisciplinaria, tarea que emprendió el CIIP desde sus inicios.

Este trabajo intenta resumir el proceso de construcción interdisciplinario de una herramienta multidimensional destinada a medir el crecimiento, desarrollo integral y bienestar infantil, SINADIBI, y presentar algunos resultados preliminares de su aplicación, ilustrando cómo la investigación académica puede contribuir en la construcción de estadísticas públicas, confiables, y sostenidas en el tiempo, y propiciar el debate sobre cómo definir los problemas de integración social de las nuevas generaciones y avanzar en el efectivo ejercicio de derechos. Para ilustrar los alcances del instrumento se exponen resultados parciales de un estudio que compara población infantil pobre residente en barrios de diferentes niveles de fragmentación territorial, seleccionando algunos de los indicadores del instrumento: condiciones de la vivienda y equipamiento del hogar no incluido en las medidas de necesidades básicas (NB), condiciones ambientales del barrio de residencia, accesibilidad a servicios, nivel de satisfacción con el lugar de residencia, indicadores de cohesión social (percepción de movilidad social) y niveles de seguridad/inseguridad alimentaria.

2 Se considera el grupo de edad que corresponde con el periodo evolutivo de la vida en el que los niños experimentan cambios decisivos y acelerados en el desarrollo, y ocurre el tránsito de la etapa prescolar a la escolar que en los países de la región tiene lugar en torno a los 7 años. 


\section{Sobre el Centro Interdisciplinario de Infancia y Pobreza (cIIP) y su punto de partida}

El CIIP comienza a funcionar a comienzos de 2010, y está integrado por diversas disciplinas que convergen en torno a un objeto de estudio e intervención compartido: los niños en su crecimiento, desarrollo integral, en aspectos del bienestar y privaciones, sus familias, el contexto micro y macro en el que viven y crecen, y los equipos e instituciones que trabajan en la implementación de políticas de infancia

Sus integrantes, con antecedentes de larga trayectoria en líneas de enseñanza e investigación vinculados con esta temática, provienen de distintas dependencias de la Universidad de la República: Depto. de Psicología Médica y Unidad de Salud Mental en Comunidad de la Facultad de Medicina (nucleados en torno al Grupo Interdisciplinario de Estudios Psicosociales -GIEP-, Banco de Datos y Depto. de Trabajo Social de la Facultad de Ciencias Sociales, Depto. de Nutrición Básica de la Escuela de Nutrición y Área de Salud de la Facultad de Psicología.

Se partía del diagnóstico, en 2009, de que si bien las políticas hacia la infancia en el país habían sido progresivamente jerarquizadas en la agenda pública uruguaya (cobertura casi total en salud y un incremento de la cobertura de programas socioeducativos para la primera infancia, entre otros), era necesario medir el impacto de esos esfuerzos y analizar en qué medida estos se traducían en mejoras del bienestar infantil. En ese momento no se disponía de un sistema de protección a la infancia integral e integrado, estatuido como política de Estado, sostenido en un soporte jurídico e institucional y con recursos de alta calidad y suficientemente estimulados como para asegurar su permanencia. Tampoco se contaba con medidas integrales de uso sistemático para evaluar la situación de los niños, y las existentes no permitían visualizar aspectos centrales vinculados con el desarrollo y bienestar infantil y con las características microsociales del entorno social, familiar y barrial. Menos aún los indicadores empleados tomaban en consideración la participación infantil.

Apoyados en dicha evidencia, uno de los propósitos del CIIP fue avanzar en la construcción de un sistema de medición integral de la situación de los niños, de aplicación sistemática, que a la vez que permitiera conocer la situación global de bienestar de la infancia, identificara la brecha de desigualdad y diferencias entre los niños. Este proceso contó con la asesoría de algunos expertos de la región.

\section{Acerca del bienestar infantil y su medición}

Siguiendo a autores como Main y Bradshaw (2012), es posible afirmar de modo genérico y desde una perspectiva de derechos, que bienestar y privación son 
dos caras de la misma moneda. Mientras el bienestar se vincula estrechamente con la satisfacción de los derechos, su contracara, la privación, se relaciona con la negación de los derechos, lo que está claramente asociado al actual concepto de pobreza infantil multidimensional. Como subrayan algunos expertos (Bradshaw et al. 2007), la discusión sobre los conceptos de bienestar de los niños ha sido útil para ampliar el debate sobre la pobreza infantil, desde una perspectiva centrada principalmente en los ingresos a una comprensión más completa de los múltiples factores que influyen en la vida de los niños.

Si bien aún débil como constructo teórico, en general se acepta que la noción de bienestar infantil debe ser multidimensional, es decir, debe: incluir aspectos físicos, emocionales y sociales; tomar en cuenta aspectos que se vinculen con la situación actual de los niños pero también con aspectos de los que dependa su desarrollo futuro; considerar medidas objetivas y también la percepción subjetiva, incluida la de los propios niños y aludir a diversos contextos en los que el niño está directamente involucrado, como el personal, familiar, amigos, escuela y comunidad o vecindario, además de los macrocontextos. (Statham, Chase 2010; CIIP 2013; Canetti et al. 2012; UNICEF 2007, 2012).

Para avanzar en la definición y operacionalización de una medida de desarrollo integral y bienestar infantil, el CIIP delimitó y desplegó cuatro grandes líneas de análisis (figura 1): i) la identificación de indicadores psicosociales robustos del desarrollo infantil, creencias y prácticas de crianza, a partir de una revisión de los instrumentos que el propio grupo había creado y que son conocidos como instrumentos GIEP (Cerutti et al. 2014), aplicados en diversos estudios de carácter nacional; ii) la búsqueda de dimensiones e indicadores de la situación nutricional, con énfasis no sólo en los componentes de la alimentación y en medidas antropométricas sino en la disponibilidad y acceso a los alimentos así como en las creencias y prácticas alimentarias, siguiendo el paradigma de "alimentación perceptiva" (OPS, 2007); iii) la identificación de indicadores de género, entre los que se destacan aquellos vinculados con el trabajo doméstico, el trabajo infantil asociado al género y las funciones de parentalidad, especialmente la función paterna, y, iv) una línea de trabajo centrada en la medida de las desigualdades, las privaciones o pobreza infantil, desde un marco multidimensional.

Algunos de los marcos teóricos discutidos incluyeron el marco normativo de los derechos del niño (ONU 1989), el marco sociológico que concibe la infancia como un sector particular de la sociedad (Qvortrup 1999, 2012; Alanen 2001; Olk 2006) y el marco del desarrollo infantil, que considera los aspectos evolutivos, concibiendo la niñez como etapa del ciclo vital y que es trabajado fundamentalmente desde la psicología, las ciencias del comportamiento y las llamadas neurociencias (Lippman 2004; Olk 2004; Stevens et al. 2005; Mustard 2005; Phillips y Shonkoff 2000; Knudsen 2004). 
Figura1. Ejes de análisis en el proceso de construcción del SINADIBI.

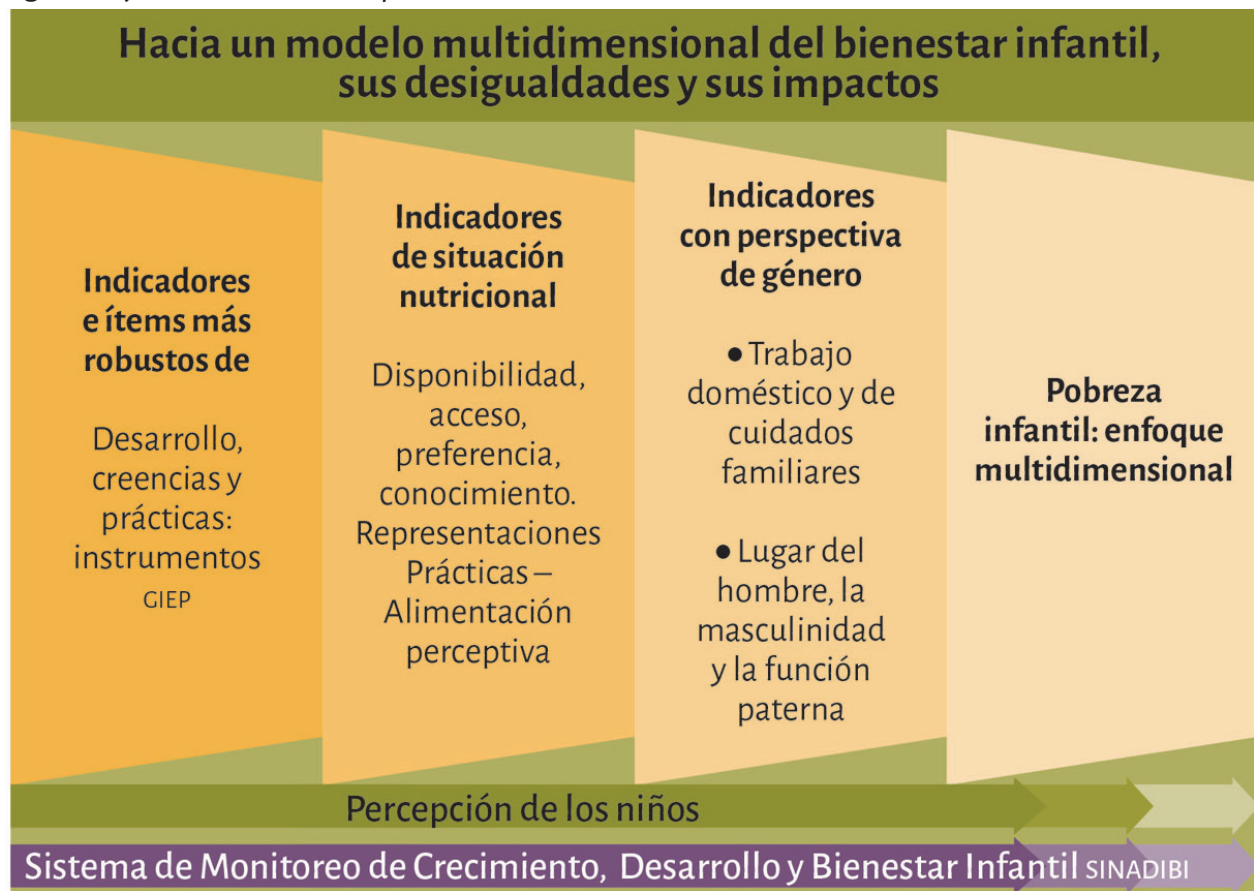

Fuente: Adaptado de (CIIP 2013, 69; Roba et al. 2013, 162).

Igualmente, se revisaron instrumentos de uso regional e internacional para consensuar qué dimensiones incluir en la definición y medición e identificar formas concretas de operacionalizar los constructos teóricos. Se revisaron, por ejemplo, los modelos de medida de pobreza y bienestar infantil de la Universidad de Bristol (Alkire et al. 2011), del CONEVAL de México (2010) empleada en Uruguay (Zacheo 2013, Informe Económico Financiero 2011), del Ministerio de Pensiones del Reino Unido (HM Government 2012), la Encuesta de Indicadores Múltiples por Conglomerados (MICS) de UNICEF (2005), también aplicada recientemente en el país (Colacce y Tennenbaum 2015) o los instrumentos que emplea el Observatorio de la Deuda Social Argentina de la UCA (ODSA 2010; Tuñón 2015, 2016).

El resultado es una herramienta que incluye un conjunto de dimensiones, componentes e indicadores multidimensionales sostenidos en los aspectos teóricos mencionados (cuadro 1).

El cuestionario se complementa con la medición del crecimiento infantil a través de datos antropométricos del niño (peso/edad, longitud-talla/edad y relación peso/talla) y la medición directa (a través de cuestionarios a adultos referentes) o indirecta (a través de la observación del niño) del desarrollo infantil. 
Cuadro 1. Dimensiones e indicadores del SINADIBI.

\begin{tabular}{|c|c|}
\hline Dimensiones & Indicadores \\
\hline \multicolumn{2}{|c|}{ Unidad de registro: Adulto de referencia del niño } \\
\hline 1. Socioeconómica & Ingresos, trabajo, educación, acceso a servicios. \\
\hline 2. Vivienda y condiciones materiales & Tenencia y condiciones de la vivienda, equipamiento. \\
\hline 3. Sistemas de cuidado & Identificación cuidadores. Percepción de la calidad educativa. \\
\hline 4. Familia & $\begin{array}{l}\text { Composición, clima familiar, estimulación cognitiva, } \\
\text { emocional y social. Autonomía, límites, tiempo libre, } \\
\text { identidad. Género. Trabajo infantil. }\end{array}$ \\
\hline 5. Salud & $\begin{array}{l}\text { Percepción del estado de salud, controles, vacunas, } \\
\text { problemas identificados, discapacidad. }\end{array}$ \\
\hline 6. Alimentación & $\begin{array}{l}\text { Seguridad alimentaria, tipo de alimentación, prácticas de } \\
\text { crianza-alimentación perceptiva. }\end{array}$ \\
\hline 7. Recursos barriales y medio ambiente & $\begin{array}{l}\text { Percepción de satisfacción y seguridad, recursos y } \\
\text { accesibilidad, medio ambiente, participación. }\end{array}$ \\
\hline 8. Cohesión social y participación & $\begin{array}{l}\text { Percepción sobre funcionamiento de la democracia, } \\
\text { seguridad laboral, confianza social, posición social percibida. }\end{array}$ \\
\hline \multicolumn{2}{|c|}{ Unidad de registro: el niño entre 3 y 7 años } \\
\hline 1. Educación & Percepción de la calidad de la institución educativa. \\
\hline 2. Seguridad & $\begin{array}{l}\text { Percepción de seguridad/inseguridad en el hogar, en el } \\
\text { barrio, en la ciudad. Conformidad con el barrio. }\end{array}$ \\
\hline 3. Participación & Actividades en las que participa, grado de satisfacción. \\
\hline 4. Salud & Percepción del estado de salud. \\
\hline
\end{tabular}

Fuente: Elaboración propia con base en (CIIP 2016).

Dentro de las características y alcances del SINADIBI destacamos que, además de abarcar dimensiones que tradicionalmente no han sido abordadas integradamente, está diseñado para niños de 0 a 7 años, con la intención de ampliarse hasta la adolescencia, es de bajo costo, accesible y puede ser empleado por técnicos no especializados pero sí entrenados en su uso. Uno de sus aspectos innovadores es el incluir la perspectiva y opinión de los propios niños en algunas de las dimensiones analizadas, a partir de los 3 años.

Permite realizar análisis comparado en la medida que incluye indicadores contenidos en otros instrumentos nacionales e internacionales. Por ejemplo, es posible construir, a partir del SINADIBI indicadores de privación infantil siguiendo los lineamientos del Observatorio de la Deuda Social Argentina (Tuñon 2015) o de Cohesión Social siguiendo criterios de la CEPAL (CEPAL 2007). 
Asimismo, la medición integral del fenómeno del desarrollo humano en la infancia temprana no solo permite una aproximación a indicadores de resultados y de procesos, sino que de modo adicional se pueden estimar y analizar las desigualdades sociales, sociorresidenciales y sociodemográficas. Ello no suele ser posible cuando se apela a registros administrativos e incluso a otras grandes encuestas o censos nacionales.

Un grupo de indicadores de interés en este instrumento, que tradicionalmente se ha manejado de manera separada del conjunto de indicadores del bienestar infantil, es el de la percepción de cohesión social (CS) por parte de las familias a las que pertenecen los niños, es decir, el grado en que las personas experimentan un sentido de pertenencia social (en diversas escalas), confían en los demás (confianza horizontal), reconocen la legitimidad de la sociedad y confían en sus instituciones (Barba Solano 2011, 71). La CS habla entonces de los distintos principios que permiten la integración social; en términos metafóricos podría decirse que la cohesión adjetiva a la integración social (Alpert 1986, 217, citado pot Barba Solano 2011, 71). Dentro de los indicadores de cohesión social incluidos en esta herramienta, se encuentra la percepción del posicionamiento y de la capacidad de movilidad social por parte de las familias a las que pertenecen los niños así como la configuración de procesos de integración y exclusión social medidos en términos de discriminación o aceptación de distintos sectores de la sociedad por parte de las familias de los niños, lo que permite identificar cuál es la población aceptada o estigmatizada por el grupo estudiado. Este conjunto de factores, sin duda, también condicionan e influyen en la calidad de vida actual y futura de los niños y en su posición social y cultural, tanto como lo hacen su acceso a la educación, a la salud, entre otras cuestiones.

Es decir que, a partir de SINADIBI, no solo se puede realizar un análisis en términos de desigualdades sociales sobre la base de atributos de los hogares que son extensibles a todos sus miembros (clima educativo, acceso a bienes y servicios, lugar de residencia, entre otros), sino que de modo adicional se cuenta con la posibilidad de realizar análisis de desigualdad en términos de aspectos subjetivos de los adultos de referencia del niño, y en relación con aspectos de tipo estructurales y objetivos.

\section{Algunas cuestiones sobre la inclusión de la opinión de los niños}

Recientemente se ha incorporado en la medición del bienestar de la infancia el denominado "bienestar subjetivo infantil", entendido como el conjunto de percepciones, evaluaciones y aspiraciones de los niños y las niñas acerca de sus propias vidas (UNICEF España 2012). Esta noción, que ha sido eje de investigaciones internacionales, se ha relacionado con la percepción de "sentirse bien", "dis- 
frutar", "estar contento", "sentirse seguro" y se ha asociado a constructos como la felicidad, la satisfacción con la vida, la satisfacción con distintos ámbitos de la vida.

La Convención de Derechos de Infancia (ONU 1989) establece, como uno de sus principios fundamentales, que la opinión de los niños debe ser tomada en cuenta en todos aquellos aspectos que les atañen, lo que ha sido concebido en términos de participación infantil. Sin duda, los niños pueden y deben ser informantes clave a la hora de analizar su bienestar y de informar y orientar las políticas públicas sobre aquello que les afecta directa o indirectamente. A pesar de ello, la mayoría de las medidas de bienestar infantil se apoyan en opiniones y percepciones de los adultos pero no incorporan la perspectiva de los niños, bajo el argumento de problemas en la confiabilidad y validez de los datos.

Si bien en el campo de la psicología la consideración directa de los niños es una práctica de larga data, empleada por ejemplo para evaluar su desarrollo, su uso en el campo de las ciencias sociales ha sido polémico y debatido. De hecho, la elaboración de indicadores sociales e índices de bienestar que contemplen su opinión es muy reciente, con la limitación, además, de que estos generalmente incluyen solo a población escolar o adolescente (Torney y Brice 1979; Melton 1980, 1983; Melton y Limber 1992; Ochaita, Espinosa y Grediaga 1994, Bradshaw et al. 2006; UNICEF-Innocenti 2007, Biggeri et al. 2006), dejando de lado por ahora a los más pequeños por las propias dificultades que implica la operacionalización de una medida de este tipo. Sin embargo, tal como lo señala Lansdown (2005), la capacidad de expresión de los niños existe desde el nacimiento aunque las formas en que lo hacen varían según la edad y momentos del desarrollo, de modo que el desafío es cómo capturar de manera objetiva dichas expresiones.

Diversos investigadores señalan que la incorporación de la opinión de los niños ha permitido mostrar los sesgos que puede tener la visión de los adultos sobre determinada situación que los afecta, por lo que promueven su inclusión en las medidas de bienestar infantil. Igualmente han señalado la importancia de analizar los datos cuidadosamente y de tomar en consideración el denominado sesgo del optimismo vital, que hace que en general los niveles de satisfacción percibida tiendan a ser altos, por lo cual su distribución no suele seguir una curva de normalidad estadística (campana de Gauss), independientemente de las condiciones sociodemográficas (UNICEF 2010; UNICEF España 2012).

Entre las recomendaciones sobre esta cuestión (Casas 2012) se destaca la inclusión de determinados indicadores psicosociales (indicadores subjetivos), como, por ejemplo: a) opiniones infantiles sobre temas que les afectan de su vida ciudadana; b) evaluaciones con ámbitos de sus vidas; c) satisfacción con los servicios que reciben; d) percepciones sobre sus derechos; e) valores que priorizan; f) actitudes y confianza hacia los adultos y las instituciones sociales. 
Contemplando estas consideraciones, el SINADIBI incorpora la perspectiva de los propios niños en varias dimensiones de análisis a través de recursos gráficos y preguntas de fácil comprensión, adaptadas a las edades a las que va dirigido el cuestionario.

\section{Algunos resultados de la aplicación del SINADIBI en el estudio del impacto de la fragmentación territorial y social en el crecimiento, desarrollo y bienestar infantil}

En 2015 se realizó un testeo de la herramienta en la ciudad de Maldonado, en un trabajo conjunto del CIIP con Facultad de Psicología y el Centro Universitario de la Región Este (CURE), de la Universidad de la República, con una muestra originalmente aleatoria de niños de 0 a 7 años que asistían a centros educativos de gestión privada de esa ciudad. Al mismo tiempo se inició un trabajo de elaboración de un sistema de información integral de la infancia con base en la Facultad de Ciencias Sociales.

En 2016 se diseñó y ejecutó el proyecto "Explorando el vínculo entre pobreza, fragmentación social y bienestar infantil en la ciudad de Montevideo", ${ }^{3}$ que permitió aplicar el instrumento como recurso metodológico para la recolección de información relacionada con el bienestar infantil.

El proyecto parte de la constatación que los cambios en los modelos productivos y de desarrollo, además de producir grandes desigualdades socioeconómicas, han impactado en los procesos de urbanización latinoamericana, generando segmentación social y una distribución territorial diferenciada y desigual en los niveles de vida, calidad ambiental y accesibilidad a los servicios. (Veiga 2010; Albagli 2008; Santos 1996). En el caso de Uruguay, estos señalamientos se evidencian claramente cuando se analiza, por ejemplo, el mapa de Montevideo y la distribución de su población según niveles de pobreza o de necesidades básicas insatisfechas (NBI), observándose cómo algunas zonas periféricas tienen alta concentración de población con NBI mientras en la costa de la capital los niveles de pobreza o NBI se reducen a sus valores mínimos (Veiga y otros 2008, 2010; Bervejillo y Lombardi 1999; MVOTMA 2007). De acuerdo con los trabajos de Veiga y otros $(2008,2010)$ la desigualdad social entre los dos extremos de la población urbana no se produce de manera dual, sino fragmentada; mientras las clases bajas continúan emigrando a la periferia de la ciudad, los sectores medios se han distribuido en todas las zonas urbanas en tanto los sectores más aventajados se

3 El proyecto estuvo a cargo de Alicia Canetti, Alejandra Girona, David Moreira, Oscar Roba y Ana Cerutti, y fue ejecutado en el marco del CIIP-EI-UDELAR, con apoyo de la Fundación Manuel Pérez de la Fac. de Medicina-UDELAR. 
concentran en la Costa este, llegando a representar el $51 \%$ de la población en dichos barrios en el 2009.

Esta segmentación y fragmentación social y territorial trae consecuencias que afectan especialmente a los sectores más pobres, quienes se ven sometidos a mecanismos y formas de exclusión que implican una reducción de la interacción entre grupos o estratos socioeconómicos distintos y una disminución de sus oportunidades para incorporar y movilizar activos que les permitan superar la pobreza y elevar su bienestar y calidad de vida (CEPAL 2003, Prieto 2008, Ballester Brage y Vecina Merchante 2011; Veiga 2010).

Dentro de los sectores más afectados por la pobreza, la segmentación y la fragmentación social, se encuentran los niños, quienes sufren el impacto de estos procesos con particular fuerza, en un momento de alta relevancia para su crecimiento y desarrollo. Al igual que ocurre en la región, este escenario urbano afecta especialmente a la infancia, por el impacto combinado de desigualdades determinadas por la pertenencia a determinados grupos de edad, la situación de ingresos de los hogares, acceso a servicios básicos, ubicación territorial, pertenencia étnica, el sistema sexo-género, entre otros factores que pueden interactuar impidiendo el ejercicio de sus derechos económicos, sociales y culturales (CEPAL, 2009). Analizado por barrios montevideanos, se observa claramente cómo los barrios con más concentración de pobreza (medida por NBI de los hogares) son los que tienen mayor concentración de niños pobres (según NBI). De esta manera, la concentración de niños con NBI podría ser empleado como un indicador proxy del grado de fragmentación territorial, concebido en términos de niveles de homogeneidad-heterogeneidad en la composición social de las zonas de residencia.

Algunos datos de este fenómeno en Montevideo muestran claramente cómo los niños más pobres se concentran en la periferia de la ciudad y cómo la estructura y los resultados escolares cambian también según las zonas de la capital que se analicen (tablas 1 y 2). Igualmente se han encontrado diferencias territoriales claras en relación a otros indicadores como la mortalidad infantil.

La literatura internacional hace referencia al beneficio que vivir en áreas de mayor intercambio social supone para el desarrollo y bienestar infantil de niños de familias pobres (Coleman 1966; Rama 1990; Maurin 2004, 48; Rich Harris 2003). En este sentido, si bien existen estudios nacionales sobre diferencias territoriales de necesidades básicas insatisfechas en la infancia (Calvo 2013), no se encuentran trabajos que analicen específicamente cuál es el impacto del grado de segmentación y fragmentación territorial en el bienestar integral de niños de hogares económicamente pobres. El presente estudio intenta aproximarse a este fenómeno comparando el crecimiento, desarrollo y bienestar infantil de niños pobres que viven en zonas con diferentes niveles de fragmentación social. 
Mapa. Organización territorial de Montevideo según CCZ.

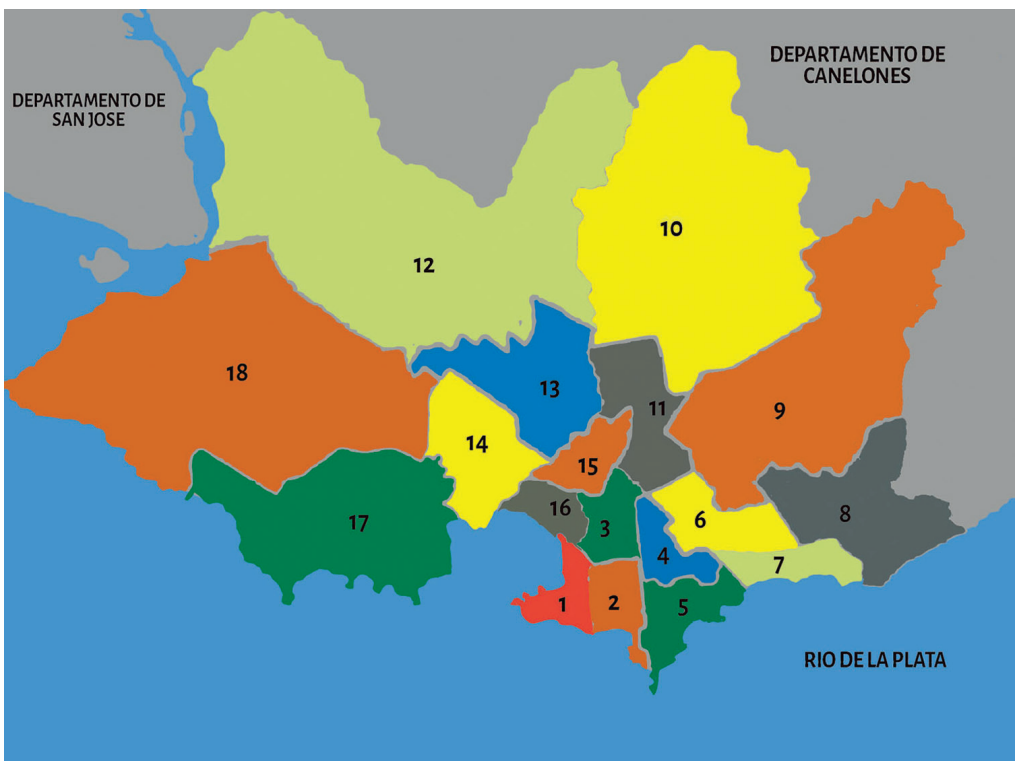

Fuente: http://www.chasque.net/vecinet/limites.htm

Tabla 1. Hogares según clima educativo.

\begin{tabular}{|l|c|c|c|c|c|c|}
\hline Zonas & Bajo $\%$ & Medio $\%$ & Alto $\%$ & Total & $\begin{array}{c}\text { Total hogares } \\
\text { particulares }\end{array}$ & $\begin{array}{c}\text { Total } \\
\text { personas }\end{array}$ \\
\hline CCZ 1 & 10.1 & 24.9 & 64.9 & 100.0 & 22,064 & 46,361 \\
\hline CCZ 2 & 4.9 & 24.6 & 70.4 & 100.0 & 47,645 & 101,225 \\
\hline CCZ 3 & 12.0 & 35.6 & 52.3 & 100.0 & 33,043 & 82,244 \\
\hline CCZ 4 & 6.5 & 19.9 & 73.6 & 100.0 & 16,002 & 37,643 \\
\hline CCZ 5 & 3.7 & 15.6 & 80.7 & 100.0 & 54,194 & 121,876 \\
\hline CCZ 6 & 21.9 & 39.1 & 39.0 & 100.0 & 22,455 & 59,030 \\
\hline CCZ 7 & 3.7 & 19.5 & 76.8 & 100.0 & 20,441 & 51,016 \\
\hline CCZ 8 & 14.3 & 26.2 & 59.5 & 100.0 & 14,222 & 43,349 \\
\hline CCZ 9 & 48.2 & 39.9 & 11.8 & 100.0 & 54,301 & 168,877 \\
\hline CCZ 10 & 53.3 & 8.0 & 8.3 & 100.0 & 14,678 & 45,944 \\
\hline CCZ 11 & 44.2 & 38.3 & 17.6 & 100.0 & 45,042 & 135,269 \\
\hline CCZ 12 & 39.7 & 36.4 & 23.9 & 100.0 & 18,519 & 57,975 \\
\hline CCZ 13 & 35.2 & 40.6 & 24.2 & 100.0 & 32,102 & 93,327 \\
\hline CCZ 14 & 35.7 & 44.5 & 18.0 & 100.0 & 28,800 & 83,112 \\
\hline CCZ 15 & 10.3 & 35.9 & 53.8 & 100.0 & 11,087 & 29.091 \\
\hline CCZ 16 & 10.1 & 32.2 & 57.7 & 100.0 & 14,372 & 37,617 \\
\hline CCZ 17 & 54.3 & 36.3 & 9.4 & 100.0 & 25,634 & 83,131 \\
\hline CCZ 18 & 45.9 & 42.3 & 11.8 & 100.0 & 12,497 & 41,668 \\
\hline Montevideo & 28.8 & 33.6 & 37.6 & 100.0 & 487,098 & $1,318,755$ \\
\hline
\end{tabular}

Fuente: Adaptada de Intendencia de Montevideo (2014,16). http://www.montevideo.gub.uy/sites/ default/files/biblioteca/informetipoporccz2014.pdf

Clima educativo: Es el promedio de años de estudio entre los miembros del hogar. Este indicador se calcula para los hogares donde vive por lo menos una persona entre 21 y 65 años de edad.

Se operacionaliza en tres niveles: bajo (cuando el promedio es menor a 9 años), medio (cuando el promedio se ubica entre 9 y 12 años) y alto (cuando el promedio es superior a los 12 años de estudio). 
Tabla 2. Años promedio de educación y rezago escolar.

\begin{tabular}{|l|c|c|}
\hline$Z_{\text {Znas }}$ & $\begin{array}{c}\text { Rezago } \\
\text { escolar }\end{array}$ & $\begin{array}{c}\text { Aĩos promedio } \\
\text { de educación }\end{array}$ \\
\hline $\mathrm{CCZ}_{1}$ & 1.8 & 13.4 \\
\hline $\mathrm{CCZ}_{2}$ & 4.6 & 14.0 \\
\hline $\mathrm{CCZ}_{3}$ & 3.5 & 12.7 \\
\hline $\mathrm{CCZ}_{4}$ & 0.9 & 14.2 \\
\hline $\mathrm{CCZ}_{5}$ & 1.9 & 15.0 \\
\hline $\mathrm{CCZ}_{6}$ & 11.2 & 11.8 \\
\hline $\mathrm{CCZ}_{7}$ & 2.8 & 14.5 \\
\hline $\mathrm{CCZ}_{8}$ & 7.6 & 13.0 \\
\hline $\mathrm{CCZ}_{9}$ & 17.7 & 9.1 \\
\hline $\mathrm{CCZ}_{10}$ & 16.7 & 8.9 \\
\hline $\mathrm{CCZ}_{11}$ & 19.2 & 9.8 \\
\hline $\mathrm{CCZ}_{12}$ & 18.1 & 10.0 \\
\hline $\mathrm{CCZ}_{13}$ & 15.9 & 10.3 \\
\hline $\mathrm{CCZ}_{14}$ & 16.8 & 9.9 \\
\hline $\mathrm{CCZ}_{15}$ & 6.2 & 12.6 \\
\hline $\mathrm{CCZ}_{16}$ & 9.8 & 13.2 \\
\hline $\mathrm{CCZ}_{17}$ & 22.5 & 8.9 \\
\hline $\mathrm{CCZ}_{18}$ & 17.8 & 9.2 \\
\hline Montevideo & 13.7 & 11.5 \\
\hline
\end{tabular}

Fuente; Intendencia de Montevideo, 2014:16 http://www.montevideo.gub.uy/sites/default/files/biblioteca/ informetipoporccz2014.pdf

Rezago escolar: refiere a la frecuencia de personas entre 8 a 15 años en los que no hay correspondencia entre los años de educación formal alcanzados (independiente del nivel) y los esperados de acuerdo a la edad. Años promedio de educación: refiere a los años promedio de educación formal para las personas entre 21 y 65 años de edad.

A partir de estos fenómenos cabe preguntarse:

A ¿Es lo mismo ser niño de un hogar económicamente pobre en el contexto de espacios sociorresidenciales socialmente heterogéneos que en el marco de espacios homogéneos en términos de su vulnerabilidad social y privaciones?

B ¿Qué diferencias genera la integración territorial con otros estratos sociales en el acceso a los derechos de educación, desarrollo y bienestar integral, en la percepción del cuidador en relación con los sistemas de apoyo sanitarios y educativos, en las prácticas de crianza, recursos barriales y ambientales y cohesión social?

Metodología: Se empleó un diseño observacional, transversal con un muestreo estratificado, con selección de las unidades por conveniencia. La estratificación se hizo con base en los datos disponibles sobre distribución de NBI según barrios, adaptada para niños menores de 6 años, lo que obligó a restringir la unidad de análisis a esta franja etaria. La selección de los estratos se justifica por el criterio de "división" de la ciudad de Montevideo a través del índice de necesidades bási- 
cas insatisfechas (NBI) elaborado a partir de los resultados del Censo 2011 ( Calvo; 2013 ) y adaptado para niños de 0 a 5 años. Así se obtiene que la ciudad de Montevideo se encuentra fuertemente segmentada en términos geográfico-económicos tanto para población general como para menores de 6 años. En este sentido se puede justificar la selección de los estratos a partir de la selección geográfica de barrios a través de criterios de NBI. Como se señaló antes, en el análisis de Montevideo se observa que mientras que un conjunto de barrios ubicados en su mayoría en la periferia montevideana presentan altos valores de población con carencias críticas (entre $40 \%$ y $60 \%$ del total de su población), todos los barrios de la costa este del departamento presentan valores muy bajos (inferiores a 14\%), lo cual evidencia una distribución territorial polarizada de las NBI.

La selección de las unidades se realizó de forma aleatoria (a través de un muestreo aleatorio simple (MAS)) dentro de los estratos.

Empleando como variable proxy de fragmentación territorial el porcentaje de niños con NBI por zonas de Montevideo, la muestra estuvo compuesta de 60 niños de hogares pobres procedentes de barrios con 60 a $70 \%$ de niños con NBI (grupo Barrio 2) y 60 niños de hogares pobres de barrios con 30 a $40 \%$ de niños con NBI (grupo Barrio 1). Los niños se distribuyeron en cuotas equivalentes por edades de 20 cada una. Se seleccionó un niño por hogar. Para medir el nivel de pobreza de los hogares se empleó una estimación del ingreso familiar, siendo seleccionados aquellos niños cuyo ingreso familiar se encontrara por debajo de la línea de pobreza y por encima de la línea de indigencia. Ambas líneas se estimaron siguiendo los criterios recomendados por el Instituto Nacional de Estadística, tomando en cuenta el número de integrantes del hogar, el valor de la canasta básica alimentaria per cápita y un coeficiente asimilable al gasto per cápita no alimentario (INE 2016, 17 y 18).

Para medir el crecimiento infantil se emplearon medidas antropométricas relevadas del carné de salud del niño y para su análisis se usaron las curvas de crecimiento según patrón de referencia de la OMS (2006); para el desarrollo infantil se utilizó un método de observación indirecta, el DIEH ${ }^{4}$ (Bedregal 2015) que interroga a los padres o cuidadores principales, y para el bienestar psicosocial el SINADIBI, que fue aplicado a alguno de los padres o cuidador principal y al niño. Se entiende por cuidador principal a la persona que habitualmente está a

4 La Evaluación para el Desarrollo Infantil Temprano para Encuesta de Hogares (DIEH) fue elaborada en Chile para ser incorporada en un módulo de desarrollo infantil en la II Encuesta Nacional de Calidad de Vida y Salud del Ministerio de Salud, realizada en el año 2006. En Uruguay este instrumento es adaptado y validado por el equipo técnico de Canelones Crece Contigo, para utilizarlo en la Encuesta de Crecimiento, Desarrollo y Salud Materna realizada en el año 2007 en Canelones (Bove, I Encuesta de Crecimiento, Desarrollo y Salud Materna en Canelones. Canelones Crece Contigo. Montevideo. UNICEF, COMUNA CANARIA, PNUD (2012)). 
cargo del cuidado del niño, que decide y realiza las acciones en relación con el desarrollo, salud y educación de los niños dentro del hogar.

\section{Algunos resultados preliminares}

En este trabajo se presentan los resultados preliminares de algunos indicadores incluidos en la encuesta. Si bien los dos grupos estudiados pertenecen a sectores de pobreza medida por ingresos y no hay diferencias estadísticamente significativas desde el punto de vista de los ingresos familiares, los resultados muestran, tal como ilustra la tabla 3, que los niños procedentes de hogares que residen en barrios con mayor fragmentación territorial (Barrio 2) presentan mayores privaciones en términos de acceso a condiciones de vida y a servicios asociados. Con respecto a indicadores de equipamiento de la casa no incluidos en la medición de NBI (menor disponibilidad de secarropas, horno microoondas, aire acondicionado y acceso a internet funcionando), la situación resulta más deficitaria en el grupo que reside en barrios de mayor concentración de pobreza. También son mayores en este grupo los problemas ambientales a nivel barrial como inundación de terrenos y calles, contaminación y quemas de basurales, pastizales y gomas.

Igualmente se observa menor accesibilidad a servicios de seguridad (comisarías) o educativos para primera infancia ( 0 a 3 años). Si bien la asistencia a servicios educativos es similar en ambos grupos, la accesibilidad a los mismos es diferente según la zona de residencia. Cerca del $43 \%$ de los hogares que viven en zonas de mayor homogeneidad social en desventaja disponen de estos servicios a más de 10 cuadras o no los tienen mientras que esto solo ocurre en el $9 \%$ de los hogares que residen en barrios con menor fragmentación socioterritorial.

En cuanto a la percepción de conformidad con el barrio de residencia, los adultos referentes difieren en los dos grupos estudiados, siendo mayor la insatisfacción entre los hogares de los niños de zonas de mayor fragmentación social (tabla 3).

No se encontraron diferencias significativas en varios indicadores de cohesión social. Los adultos referentes o cuidadores principales en ambos grupos comparten la percepción sobre su movilidad social ascendente o descendente con respecto a sus propios padres y a la que tendrán sus hijos en el futuro con respecto a ellos ("Imagínese una escala de 10 lugares, en que en el 1 se ubican las personas más pobres y en el 10 se ubican las más ricas... ¿Dónde se ubicaría Ud., dónde ubicaría a sus padres y dónde a sus hijos en el futuro?"). Es interesante que ninguno de los niños conviva con adultos referentes que se perciban a sí mismos en una mejor posición económica que sus propios padres (abuelos del niño) y cerca de un tercio en ambos grupos percibe estar peor. En cambio más de la mitad de los padres o adultos referentes entrevistados en ambos grupos aspira a que sus hijos estén mejor que ellos y ninguno se imagina que sus 
Tabla 3. Características del acceso a bienes y servicios y satisfacción con el lugar de residencia según barrios.

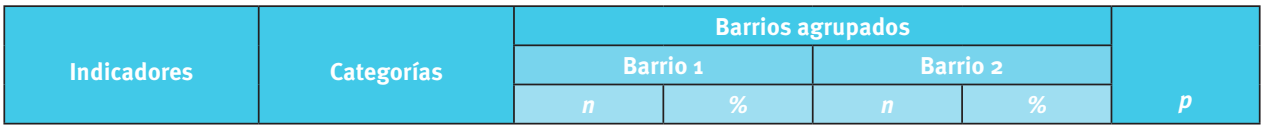

\section{Condiciones vivienda no incluidas en NBI}

\begin{tabular}{|c|c|c|c|c|c|c|}
\hline \multirow{2}{*}{ Escasa ventilación } & No & 53 & 88.3 & 46 & 76.7 & \multirow{2}{*}{0.093} \\
\hline & Sí & 7 & 11.7 & 14 & 23.3 & \\
\hline \multirow{2}{*}{$\begin{array}{c}\text { Se inunda con la } \\
\text { lluvia }\end{array}$} & No & 52 & 86.7 & 44 & 73.3 & \multirow{2}{*}{0.068} \\
\hline & Sí & 8 & $13 \cdot 3$ & 16 & 26.7 & \\
\hline
\end{tabular}

\section{Equipamiento no básico de la vivienda}

\begin{tabular}{|c|c|c|c|c|c|c|}
\hline \multirow{2}{*}{ Secarropas } & Sí & 8 & 13.3 & 7 & 5 & \multirow{2}{*}{ NS } \\
\hline & No & 52 & 86.7 & 53 & 95 & \\
\hline \multirow{2}{*}{ Horno microondas } & Sí & 46 & 76.7 & 30 & 50 & \multirow{2}{*}{0,004} \\
\hline & No & 14 & 23.3 & 30 & 50 & \\
\hline \multirow{2}{*}{ Aire acondicionado } & Sí & 19 & 31.7 & 12 & 20 & \multirow{2}{*}{ NS } \\
\hline & No & 41 & 68.3 & 48 & 80 & \\
\hline \multirow{2}{*}{ Acceso a Internet } & Sí & 51 & 85 & 34 & 56.7 & \multirow[b]{2}{*}{0,001} \\
\hline & No & 9 & 15 & 26 & 43.3 & \\
\hline
\end{tabular}

\section{Condiciones ambientales de zona de residencia}

\begin{tabular}{|c|c|c|c|c|c|c|}
\hline \multirow{2}{*}{$\begin{array}{l}\text { Terrenos y calles } \\
\text { inundadas }\end{array}$} & Sí & 5 & 8.3 & 23 & 38.3 & \multirow[t]{2}{*}{0.000} \\
\hline & No & 55 & 91.7 & 37 & 61.7 & \\
\hline \multirow{2}{*}{ Terreno contaminado } & Sí & 4 & 6.7 & 11 & 18.3 & \multirow[t]{2}{*}{0.050} \\
\hline & No & 56 & 93.3 & 49 & 81.7 & \\
\hline \multirow{2}{*}{$\begin{array}{l}\text { Quema de basura, } \\
\text { pastizales, gomas }\end{array}$} & Sí & 6 & 10 & 23 & 38.3 & \multirow[t]{2}{*}{0.000} \\
\hline & No & 54 & 90 & 37 & 61.7 & \\
\hline \multirow{2}{*}{$\begin{array}{c}\text { Al menos un corte de } \\
\text { luz en los últimos } \\
6 \text { meses }\end{array}$} & Sí & 23 & 38.3 & 37 & 61.7 & \multirow[t]{2}{*}{0.017} \\
\hline & No & 37 & 61.7 & 23 & 38.3 & \\
\hline
\end{tabular}

\section{Acceso a servicios}

\begin{tabular}{|c|c|c|c|c|c|c|}
\hline \multirow{4}{*}{ Acceso a Comisaría } & Menos de cinco & 14 & 23.7 & 0 & 0 & \multirow{4}{*}{0,000} \\
\hline & 5 a 10 & 14 & 23.7 & 7 & 11.7 & \\
\hline & 10 & 27 & 45.8 & 43 & 71.7 & \\
\hline & No hay & 4 & 6.8 & 10 & 16.7 & \\
\hline \multirow{4}{*}{$\begin{array}{l}\text { Acceso a jardín para } \\
\text { menores de } 3 \text { años } \\
\text { (en cuadras) }\end{array}$} & Menos de cinco & 33 & 57.9 & 14 & 24.1 & \multirow{4}{*}{0,000} \\
\hline & 5 a 10 & 19 & 33.3 & 19 & 32.8 & \\
\hline & 10 & 3 & $5 \cdot 3$ & 17 & 29.3 & \\
\hline & No hay & 2 & 3.5 & 8 & 13.8 & \\
\hline \multirow{4}{*}{$\begin{array}{l}\text { Acceso a } \\
\text { Cajero automático } \\
\text { (en cuadras) }\end{array}$} & Menos de cinco & 17 & 28.8 & 8 & 13.3 & \multirow{4}{*}{0,000} \\
\hline & 5 a 10 & 14 & 23.7 & 26 & 43.3 & \\
\hline & 10 & 23 & 39 & 24 & 40 & \\
\hline & No hay & 5 & 8.5 & 2 & 3.3 & \\
\hline \multicolumn{7}{|c|}{ Satisfacción con el lugar de residencia } \\
\hline \multirow{2}{*}{$\begin{array}{l}\text { Percepción sobre } \\
\text { el barrio }\end{array}$} & Satisfecho & 56 & 97 & 46 & 79 & \multirow{2}{*}{0,004} \\
\hline & Insatisfecho & 2 & 3 & 12 & 21 & \\
\hline
\end{tabular}

Fuente: CIIP, 2017. Elaboración propia. 
hijos estarán en una peor condición (lo que es probablemente una expresión de deseo respecto al futuro social de sus hijos) (figura 2).

Uruguay tiene poca experiencia en el uso de la medición de la seguridad alimentaria y nutricional (SAN) de los hogares a través de la Escala Latinoamericana y Caribeña de Seguridad Alimentaria (ELCSA) (Comité Científico de la ELCSA-FAO 2012), la cual mide la percepción de los hogares sobre su situación de SAN. En el caso de la Encuesta Alimentación y Bienestar realizada por el Núcleo Interdisciplinario Alimentación y Bienestar de la UDELAR-INDA en 2014 (UDELAR, INDA 2015), aplicada a hogares de Montevideo y área metropolitana, se observó que para el total de hogares, el 60.9 \% presentó seguridad alimentaria, el resto se distribuyó entre un $24.7 \%$ para InSAN leve, $7.3 \%$ para InSAN moderada y $7.1 \%$ para InSAN grave. Se destacó en este estudio que la prevalencia de inseguridad alimentaria fue mayor en los hogares integrados por adultos y menores de 18 años, lo que mostraba que la InSAN era mucho más prevalente en hogares con menores a su cargo.

El otro estudio corresponde a la Primera Encuesta de Nutrición, Desarrollo Infantil y Salud (ENDIS), relevada en los años 2012 y 2013, la cual mostró que el 57\% de los hogares con niños menores de 4 años se encontraba en situación de seguri-

Figura 2. Percepción de movilidad social de los padres/cuidador principal con respecto a sus propios padres y a sus hijos (\% hogares).

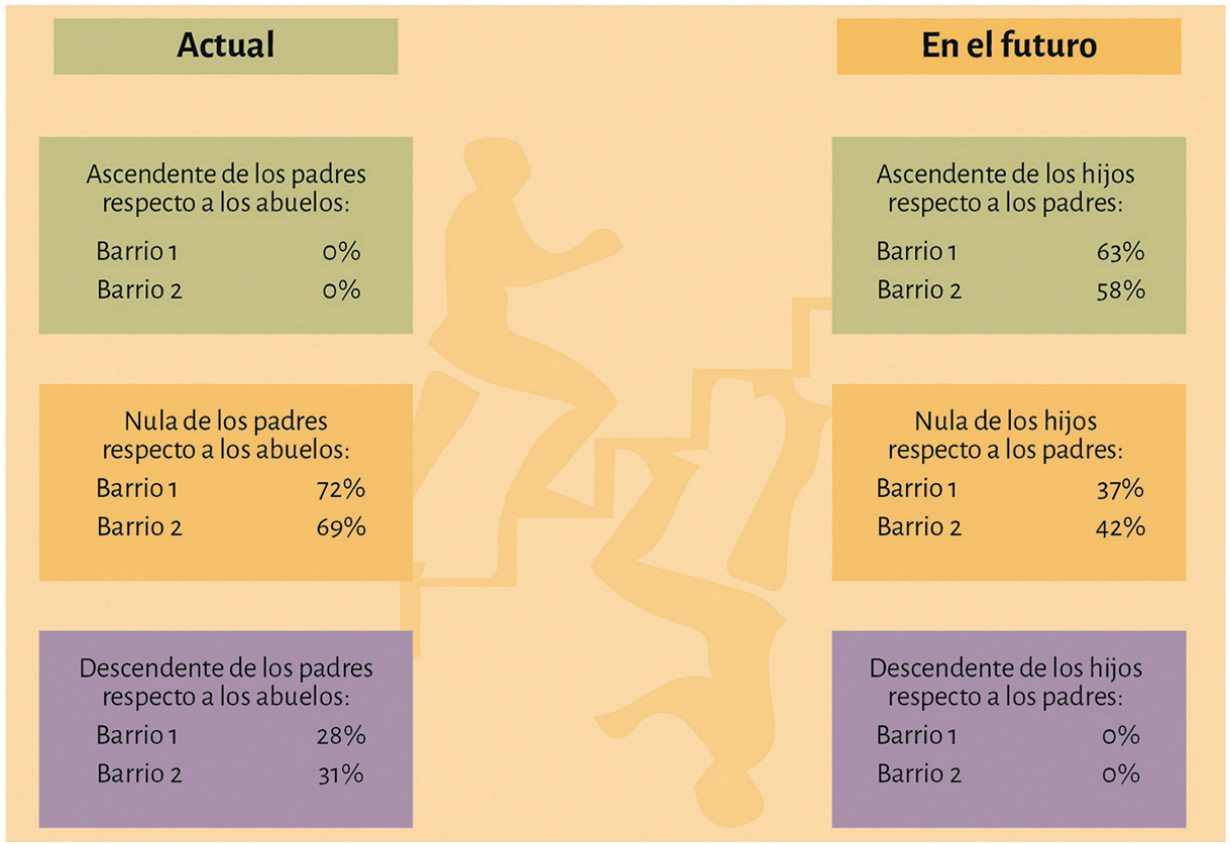

Fuente: Elaboración propia, 2017. 
dad alimentaria, mientras que el 28\% presenta inseguridad leve, el 9\% inseguridad moderada y el $4.3 \%$ inseguridad grave. ${ }^{5}$ (UDELAR, INE, UCC-MIDES 2015).

El presente estudio muestra con datos más actualizados, que los niños que viven en barrios de mayor fragmentación territorial pertenecen a hogares con mayor niveles de inseguridad alimentaria, alcanzando cifras de $63.7 \%$, mientras que en los hogares de territorios con menor fragmentación social la InSAN alcanza al 38.3 \% (similar a la observada en población general en los dos estudios anteriores). Para ambos grupos la InSAN está concentrada en la categoría leve, pero al observar la categoría más grave de inseguridad, la prevalencia en los hogares de niños que residen en zonas de mayor fragmentación es el doble que en el otro grupo (tabla 4)

Si bien los estudios no son comparables, las cifras obtenidas por el CIIP muestran que el problema del hambre no ha sido aún resuelto, se agudiza en la pobreza y se convierte en un gran desafío para Uruguay de cara a los próximos años.

Los datos preliminares de este estudio analizados hasta el momento confirman la hipótesis acerca de que, aún en las mismas condiciones de pobreza medida por ingresos, el grado de fragmentación territorial afecta diversos aspectos relacionados con los niveles de vida, calidad ambiental y accesibilidad a los servicios por parte de los niños y sus familias, reduciendo las oportunidades para incorporar y movilizar activos que contribuyan a superar la pobreza y elevar el bienestar y calidad de vida.

\section{A modo de cierre}

Existe amplio consenso en la necesidad de promover el bienestar de la infancia, con suficiente evidencia de los costos individuales y sociales atribuibles a su pos-

Tabla 4. Niveles de seguridad alimentaria según barrios.

\begin{tabular}{|c|c|c|c|c|c|c|}
\hline \multirow{3}{*}{ Indicadores } & \multirow{3}{*}{ Categorias } & \multicolumn{4}{|c|}{ Barrios agrupados } & \multirow[b]{3}{*}{$p$} \\
\hline & & \multicolumn{2}{|c|}{ Barrio 1} & \multicolumn{2}{|c|}{ Barrio 2} & \\
\hline & & $n$ & $\%$ & $n$ & $\%$ & \\
\hline \multirow{4}{*}{$\begin{array}{l}\text { Seguridad } \\
\text { alimentaria } \\
\text { según ELCSA }\end{array}$} & Seguro & 37 & 61.7 & 21 & 36.2 & \multirow{4}{*}{0.04} \\
\hline & Inseguridad leve & 17 & 28.3 & 25 & 43.1 & \\
\hline & Inseguridad moderada & 5 & 8.3 & 10 & 17.2 & \\
\hline & Inseguridad severa & 1 & 1.7 & 2 & 3.4 & \\
\hline
\end{tabular}

Fuente: CIIP, 2017. Elaboración propia.

5 El Observatorio de la Deuda Social Argentina estimó para el caso de la Argentina urbana en 2015 un $20.8 \%$ de niños en hogares con inseguridad alimentaria y $8.2 \%$ en situación grave. La evolución 2010-2015 marca una merma de apenas 1.2 p.p. (Tuñón 2015). 
tergación. Uruguay ha hecho esfuerzos para profundizar en políticas públicas especialmente orientadas a la población infantil, lo que a su vez plantea la urgente necesidad de medir su impacto y analizar en qué medida estos se traducen en avances en la protección y promoción del bienestar de dicha población. La medición del bienestar infantil es un área de estudio relativamente nueva, de gran complejidad, con muchas definiciones sobre cuáles son sus principales dimensiones y sus formas de operacionalizarlas. La propuesta de desarrollo de una herramienta de medición multidimensional del crecimiento, desarrollo y bienestar de la infancia realizada por el Centro Interdisciplinario de Infancia y Pobreza aspira contribuir a cubrir un "vacío" que tienen los Estados de la región en términos de garantizar el seguimiento, monitoreo y evaluación de las políticas públicas destinadas a la protección de los derechos de las niñas y los niños. Al mismo tiempo se trata de aportar, desde la investigación académica, a la construcción de estadísticas públicas, confiables, y sostenidas en el tiempo, que sirvan para rendición de cuentas a la ciudadanía y para orientar hacia un uso efectivo de la inversión pública.

El desafío es que esta herramienta se integre a un sistema único nacional de evaluación del estado de la infancia en Uruguay, aplicado de manera periódica y continua y que pueda ser ampliado progresivamente a otros grupos etarios, integrando siempre la opinión de los niños como fuente de información. Por otra parte, se aspira a aplicarlo en el marco de propuestas colaborativas a nivel regional, ofreciendo una mirada más amplia y comparada de la situación de la infancia en América Latina. ld

\section{Referencias}

Alanen, L. "Childhood as a generational condition.» En L. Alanen y B. Mayall (eds.), Conceptualizing child - adult relations. Londres: Falmer, 2001, 6-49.

Albagli, A. Los procesos de fragmentación socio-territorial a nivel local: causas y efectos. Cómo afectan la identidad urbana. Un estudio de caso. Informe Integrar, $\mathrm{N}^{\circ}$ 49-junio de 2008. Instituto de Integración Latinoamericana, Facultad de Ciencias Jurídicas y Sociales, Universidad Nacional de La Plata (IIL-FCJSUNLP. http://www.iil.jursoc.unlp.edu.ar/textos/informe/integrar49.pdf

Alkire, Sabina; José Manuel Roche; María Emma Santos y Suman Seth. Uruguay Country Briefing. Oxford Poverty \& Human Development Initiative (OPHI) Multidimensional Poverty Index Country Briefing Series. UK: University of Oxford, 2011. www.ophi.org.uk/policy/multidimensional-poverty-index/mpicountry-briefings/

Alpert, H. Durkheim. México: FCE, 1986, 217.

Ballester Brage, Lluís; Merchante, Carlos Vecina. «Intervención comunitaria, di- 
versidad y complejidad social: el problema de la segregación social en la escuela.» Prisma Social: revista de Ciencias sociales, 6, 2011, 1-29.

Barba Solano, C. «Revisión teórica del concepto de cohesión social: hacia una perspectiva normativa para América Latina.» En Perspectivas críticas sobre la cohesión social: Desigualdad y tentativas fallidas de integración social en América Latina. Buenos Aire: CLACSO, 201 1, 67-86.

Bedregal, P., Hernández, V., Yeomans, H., Molina, H. «Validez concurrente de un instrumento simple de evaluación del desarrollo infantil temprano para encuestas de hogares.» Rev. Med. Chile, 141, 2013, 409-410.

Biggeri, M., Libanora, R., Mariani, S. y Menchini, L. «Children conceptualizing their capabilities: Results of a survey conducted during the First Children's World Congress on Child Labour.» Journal of Human Development, 7(1) 2006, 59-83, ISSN 1464-9888.

Bradshaw, J., Hoelscher, P. y Richardson, D. «Comparing child well-being in OECD countries: Concepts and methods.» Innocenti Working Paper, No. 2006-03. Florence: UNICEF Innocenti, 2007.

—. "An index of child well-being in the European Union.» Social Indicators Research, 80, 2006, 133-167.

Calvo, Juan José et al. Atlas sociodemográfico y de la desigualdad del Uruguay. Las necesidades básicas insatisfechas a partir de los censos 2011. Instituto Nacional de Estadística, Universidad de la República, Facultad de Ciencias Sociales, Unidad Multidisciplinaria. Montevideo: Trilce, 2013.

Canetti, A., Schwartzmann, L.; De Martino, Mónica; Bagnato, Ma. José; Roba, Óscar; Girona, Alejandra; Cerutti, Ana; Espasandín, Cecilia y Álvarez, Magdalena. "Pobreza infantil: conceptos e indicadores" Revista Digital Universitaria, 13, 5, 2012.

Casas, F. y Bello, A. (coords.), González M., Aligué M., Bertrán I., Montserrat, C., Navarro, D., González-Bueno Uribe, G., von Bredow, M. Calidad de vida y bienestar infantil subjetivo en España ¿Qué afecta al bienestar de niños y niñas españoles de $1^{\circ}$ de ESO? Universidad de Girona-Unicef. Madrid: UNICEF, 2012.

CEPAL, EUROPEAID. Un sistema de indicadores para el seguimiento de la cohesión social en América Latina. Santiago de Chile: CEPAL, 2007.

CEPAL. Pobreza infantil, desigualdad y ciudadanía. Iniciativa CEPAL/UNICEF en América Latina y el Caribe, 2009.

CEPAL-UNICEF. "Guía para estimar la pobreza infantil.», 2012. http://dds.cepal. org/infancia/guia-para-estimar-la-pobreza-infantil

CEPAL-Universidad Estado Michigan. Capital social y reducción de la pobreza en América Latina y el Caribe: en busca de un nuevo paradigma, vol. 71. United Nations Publications, 2003.

Cerutti, A., Canetti, A., Scwartzmann, L. Desarrollo psicomotor y prácticas de cri- 
anza: Su evaluación instrumentos de tamizaje del Grupo Interdisciplinario de Estudios Psicosociales. (GIEP). CIIP-EI-Udelar. Montevideo: Tradinco, 2014.

CIIP. «Crecimiento, desarrollo y bienestar infantil en condiciones de pobreza. Teorías, modelos e indicadores.» En Canetti, A. y Schwartzmann, L. (coords.), Modelos e indicadores de desarrollo y bienestar infantil, Seminario (integrantes: De Martino, M., Bagnato, M., J., Roba, O., Girona, A., Cerutti, A., Espasandín, C. y Álvarez, M.). Montevideo: Tradinco-Centro Interdisciplinario de Infancia y Pobreza (CIIP)-Espacio Interdisciplinario-UDELAR, 2013.

Colacce, M., Tennenbaum, V. Pobreza y privaciones múltiples en la infancia en Uruguay, vol. 115. Montevideo: CEPAL-UNICEF, 2016.

Coleman, James Samuel. Equality of educational opportunity. Washington, DC: US Department of Health, Education, and Welfare, Office of Education, 1966. Comité Científico de La ELCSA-FAO. Escala Latinoamericana y Caribeña de Seguridad Alimentaria (ELCSA): Manual de uso y aplicaciones. Roma: FAO, 2012.

Coneval (Consejo Nacional de Evaluación de la Política de Desarrollo Social). Metodología para la medición multidimensional de la pobreza en México. Mexico, 2010. http://www.coneval.org.mx/Informes/Coordinacion/INFORMES_Y_ PUBLICACIONES_PDF/Metodologia_Multidimensional_web.pdf

Díaz Gorfinkiel, Magdalena. «Más que cuidadoras: ciudadanas de nuevas dinámicas sociales.» Prisma Social: revista de Ciencias sociales, 5, 2010, 1-28. http:// www.coneval.gob.mx/cmsconeval/rw/resource/Metodologia_Medicion_ Multidimensional.pdfConvención Internacional de los Derechos del Niño HM Government. Measuring child poverty: A consultation on better measures of child poverty. Presentado al Parlamento por el Secretary of State for Work and Pensions by Command of Her Majesty. Noviembre, 2012. UK: UK: Stationery Office Limited on behalf of the Controller of Her Majesty's Stationery Office, 2012. http://www.officialdocuments.gov.uk/document/cm84/8483/8483.pdf http://redproteccionsocial.org/sites/default/files/uruguay_presentacion_ pm_lz.pptx

http://www.mides.gub.uy/innovaportal/v/44017/3/innova.front/primeraencuesta-nacional

Informe Económico-Financiero de la Rendición de Cuentas y Balance de Ejecución Presupuestal de 2011. http://www0.parlamento.gub.uy/htmlstat/cepre/ rendicion2011/informe.pdf

Instituto Nacional de Estadística. Estimación de la pobreza por el Método del Ingreso, 2015. Montevideo: INE, 2016, 17-18.

Intendencia de Montevideo. Información Física y Sociodemográfica por Centro Comunal Zonal Fuente: Fase 1 censo 2004, censo 2011. ECH 2014, Montevideo: IM, abril, 2014. 
Knudsen, E. I. «Sensitive periods in the development of the brain and behavior.» Journal of Cognitive Neuroscience, 16, 2004, 1412-1425.

Lansdown, G. «La evolución de las facultades del niño.» Florencia: Centro Innocenti UNICEF, 2005. http://www.unicef-irc.org/publications/pdf/EVOLVING-E. pdf

Lippman, L. Indicators of child, family and community connections. Washington, D.C.: Office of the Assistant Secretary for Planning and Evaluation, US Department of Health and Human Services.

Lombardi, M. Y., Bervejillo, F. Globalización, integración y expansión metropolitana en Montevideo. UCUDAL. Conferencia presentada en el V Seminario Internacional de la RII, Toluca, México, septiembre, 1999.

Main, G., Bradshaw, J. «A child material deprivation index.» Child Ind Res, 2012. DOI 10.1007/s12187-012-9145-7.

Maurin, E. Le ghetto français. Enquête sur le séparatisme social. París: Seuil, $2004,48$.

Melton, G. B. «Children's concepts of their rights.» Journal of Clinical Child Psychology, 9, 1980, 186-190.

- Child advocacy: Psychological issues and interventions. Nueva York: Plenum Press, 1983.

— y Limber, S. «What children's rights mean to children: Children's own views.». En M. Freeman y P. Veerman (eds.), Ideologies of children's rights. Dordrecht, Países Bajos: Martinus Nijhoff, 1992, 167-187.

Mustard, J. F. Child development: An experience-based brain development: The scientific underpinnings of the importance of early child development in a globalized world. Canadian Institute for Advanced Research. Informe presentado al International Symposium on early child development. Septiembre, 2005, 27-29. http://www.brookings.edu/views/papers/200602mustard.htm mvotma. El Libro Blanco del Área Metropolitana. Montevideo: Programa Agenda Metropolitana, 2007.

Myers, R. Reflexiones sobre indicadores del desarrollo de niños menores de seis años, teleconferencia, Memorias del Foro Primera Infancia y Desarrollo. El Desafío de la Década. Bogotá: 21 Instituto Colombiano de Bienestar FamiliarICBF Alcaldía Mayor de Bogotá D.C. Departamento Administrativo de Bienestar Social-DABS, Save the Children, Reino Unido, UNICEF, Centro Internacional de Educación y Desarrollo Humano-CINDE. 2003, 157-174.

Observatorio de la Deuda Social Argentina (ODSA). «Barómetro de la deuda social de la infancia.» La deuda social con la niñez y adolescencia: magnitud, evolución y perfiles, 4. Argentina: Universidad Católica Argentina, 2010.

Ochaita, E. Espinosa, M. A. y Grediaga, M. C. «¿Cómo entienden los niños el derecho a la igualdad?» Infancia y Sociedad, 1994, 27-28 y 61-76. 
Olk, T. «Welfare states and generational order.» En H. Wintersberger, L. Alanen, T. Olk y J. Ovortrup (eds.), Childhood, generational order and the welfare state. Exploring children's social and economic welfare. Odense: University of Southern Denmark Press, 2006, 59-90

—. "German children's welfare between economy and ideology.» En A. M. Jensen, A. Ben-Arieh, C. Conti, D. Kutsar, M. N. G. Phádraig y H.W. Nielsen (eds.), Children's welfare in an ageing Europe, vol. 2. Oslo: Norwegian Centre for Child Research, 2004.

OMS. Who Child Growth Standards. Ginebra: World Health Organization, 2006.

ONU. Oficina del Alto Comisionado para los Derechos Humanos. Convención sobre los Derechos del Niño. Adoptada y abierta a la firma y ratificación por la Asamblea General, en su resolución 44/25, del 20 de noviembre de 1989. http://www2.ohchr.org/spanish/law/crc.htm

Organización Panamericana de la Salud. Principios de orientación para la alimentación del niño no amamantado entre los 6 y los 24 meses de edad. Washington, D.C.: OPS, 2007.

Phillips, Deborah A. y Jack, P. Shonkoff (eds.). From neurons to neighborhoods: The science of early childhood development. Washington, DC, USA: National Academies Press, 2000.

Prieto, M. B. «Fragmentación socio-territorial y calidad de vida urbana en Bahía Blanca.» Geograficando, año 4, no. 4, 2008, 193-214.

Qvortrup, J. Conferencia dictada en el V Congreso Mundial sobre Infancia. San Juan, Argentina, 2012 https://www.youtube.com/watch?v=be-EV8Fq6q0

—. "The meaning of child's standard of living.» En A. B. Andrews y N. H. Kaufman (eds.), Implementing the U.N. Convention on the Rights of the Child: A standard of living adequate for development. Westport, CT: Praeger, 1999. Rama, G. Qué aprenden y quiénes aprenden en las escuelas de Uruguay: los contextos sociales e institucionales de éxitos y fracasos. Uruguay CEPAL. Oficina De Montevideo, 1990.

Rich Harris, J. El mito de la educación. Barcelona: Debolsillo, 2003.

Roba, O., Cerutti, A., Canetti, A., Parafita, D. y Duarte, D. «4.1 Pobreza infantil. Conceptos, dimensiones y medidas.» En Estrategias regionales de medición de pobreza en niñez. Editado por GT Infancia, Desigualdades y Pobreza / Equidad para la Infancia, Bs As: Equidad para la infancia-UNSM, 2013, 162. http://www.equidadparalainfancia.org/wp-content/uploads/2013/09/ PUBLICACI\%C3\%93N-FINAL-GT-2013.pdf

Santos, M. Metamorfosis del espacio habitado. Barcelona: Oikos-Tau, 1996.

Statham, June y Elaine Chase. Childhood wellbeing: A brief overview. Loughborough, UK: Childhood Wellbeing Research Centre, 2010.

Stevens et al. Focus on families: Reinforcing the importance of family. Families with 
dependent children - Successful outcomes project. Report on literature review and focus groups. Wellington, Nueva Zelanda: Families Commission, 2005. http://www.familiescommission.govt.nz/download/focus-on-families.pdf

Torney, J. y Brice, P. Children's concepts of human rights and social cognition. Investigación presentada en la American Psychological Association. Nueva York. Washington, DC: National Council for the Social Sciences, 1979. [Citado en Torney-Purta, J. «Socialization and human rights research: Implications for teachers.» En Branson, M. S. y Torney-Purta (eds.), International human rights, society, and the schools, 1982, 35-48].

Tuñón, I., Poy, S., Coll, A. Pobreza infantil en las ciudades de la Argentina 20102014: Diferentes mediciones de la pobreza infantil y una propuesta multidimensional desde un enfoque de derechos. Ianina Tuñón; Santiago Poy; Agustina Coll.-la edición especial-Ciudad Autónoma de Buenos Aires: Educa, 2015.

—_, Ianina y Poy, Santiago. Las múltiples dimensiones de la pobreza infantil. Incidencia, evolución y principales determinantes. Periodo 2010-2015, la ed. Ciudad Autónoma de Buenos Aires: Educa, 2016.

UDELAR, INDA, Encuesta Alimentación y Bienestar (Núcleo Interdisciplinario Alimentación y Bienestar Informe final. Montevideo, 2015.

- INE, UCC-MIDES. Primera Encuesta Nacional de Salud, Nutrición y Desarrollo Infantil (ENDIS). Montevideo, 2015. http://www.mides.gub.uy/innovaportal/ file/44017/1/informe-ucc-endis.pdf

UNICEF (Gabriel González-Bueno, Maria von Bredow, Carlos Becedóniz). Propuesta de un sistema de indicadores sobre bienestar infantil en España. Madrid: UNICEF, 2010.

—_ España. El bienestar infantil desde el punto de vista de los niños: ¿qué afecta el bienestar de niños y niñas de $1^{\circ}$ de ESO en España? Madrid: UNICEF España, 2012.

- Pobreza infantil en perspectiva: un panorama del bienestar infantil en los países ricos, Innocenti Report Card 7, Centro de Investigaciones Innocenti de UNICEF, Florencia. (c) Fondo de las Naciones Unidas para la Infancia, 2007.

- Innocenti Research Centre. Measuring child poverty: New league tables of child poverty in the world's rich countries. Innocenti Report Card 10, UNICEF Innocenti Research Centre. Florence, United Nations Children's Fund (UNICEF), 2012. http://www.unicefirc.org/publications/pdf/rc10_eng.pdf

—. Dar seguimiento a la situación de niños, niñas y mujeres. Manual para la encuesta de indicadores múltiples por conglomerados. Nueva York: UNICEF, División de Políticas y Planificación, 2005.

Veiga, D. Estructura social y ciudades en el Uruguay: tendencias recientes. Montevideo: Facultad de Ciencias Sociales-CSIC, UDELAR, 2010. 
—. «Fragmentación socioterritorial y desigualdades en el Área Metropolitana de Montevideo.» PAMPA, 1, 4, 2008, 11-36.

—_ Ana Laura Rivoir. «Fragmentación socioeconómica y segregación urbana en Montevideo.» Ciudad y Territorio-Estudios Territoriales, 158, 2008.

Zacheo, L. Avances para la medición multidimensional de la pobreza en Uruguay desde un enfoque de derechos. Taller sobre índices de pobreza multidimensional: buenas prácticas y lecciones aprendidas de Colombia, México y Uruguay. Dirección Nacional de Evaluación y Monitoreo (DINEM), Ministerio de Desarrollo Social de Uruguay-MIDES. Bogotá, 2013. 
